\title{
Status of DTPA-Extractable Cationic Micronutrients and Physio Chemical Properties of Geo Referenced Soils Under Irrigated and Rainfed Ecosystems of Vellore District in Tamilnadu, India
}

\author{
Purma Soniya*, D. Muthumanickam, M.R. Reshma and N. Jayasoorian \\ Department of Soil Science and Agricultural Chemistry, \\ Tamil Nadu Agricultural University, India
}

*Corresponding author

\begin{abstract}
A B S T R A C T
\section{Keywords}

DTPA - extractable micronutrients, Soil physical and chemical properties, Irrigated ecosystem, rainfed ecosystem, GIS (Geographical Information System), GPS (Geographical positioning System)

Article Info

Accepted:

12 May 2019

Available Online:

10 June 2019

The DTPA extractable cationic micronutrient and some important soil physico-chemical properties were investigated in two ecosystems namely irrigated and rainfed conditions of Vellore district of Tamil Nadu using GPS and GIS techniques. Considering the critical limits of soil micronutrients, all soil associations were not adequately supplied with DTPA extractable micronutrients in both the ecosystems. Irrespective of land use, the DTPA extractable cationic micronutrients were positively related with organic carbon content and negatively related to calcium carbonate content. The soils under rainfed condition possessed lesser values of all the nutrient availability than irrigated soil environment except for zinc which nutrient content is equal. Deficient soil samples for manganese and copper were found in some blocks under irrigated conditions and all the blocks for zinc deficiency except Gudiyattam block and for iron deficiency none of the block is deficient. In rainfed ecosystem, iron and manganese deficiency is noticed in some blocks and zinc deficiency in all the blocks and copper deficiency is seen in all the blocks except Alangayan block.
\end{abstract}

\section{Introduction}

Indian agriculture during the past 50 years has achieved a fourfold growth in food production by adopting modern agricultural practices. However, intensive cultivation of high yielding varieties, increased use of NPK fertilizers free from secondary and micronutrients, decreased use of organic manures and lack of crop residue recycling have led to depletion of native nutrient fertility and resulted in wide spread deficiencies of secondary and micronutrients. The deficiency may either be primarily due to their low contents or secondarily by soil factor that reduce the availability (Sharma and Chaudhary, 2007). Micronutrients are as essential as macronutrients but required in smaller quantities by plants. The extent of micronutrient deficiency varies not only in different states and districts but also in different blocks within the district. Micronutrient deficiencies is the one of the main causes for low yield or crop yield 
decline in irrigated (Katyal and Rattan, 2003) and rainfed cropping system (Rego et al., 2007). On the basis of computation of exhaustion period of micronutrient reserve under different cropping system soil zinc is the most limiting micronutrient (Rattan et al., 2009).

Besides soil characteristics, land use pattern also plays a vital role in governing the nutrient dynamics and fertility of soils (Venkatesh et al., 2003). Due to continuous cultivation, soils under a particular land use system may affect physico-chemical properties which may modify DTPAextractable micronutrients content and their availability to crops. So, analysis of these properties along with micronutrient status of different land use systems may have significant importance. Scanty information is available on status of cationic micronutrients under different ecosystems in the Vellore district of Tamil Nadu. An attempt has, therefore, been made to generate information regarding the DTPA- extractable $\mathrm{Fe}, \mathrm{Zn}, \mathrm{Mn}$ and $\mathrm{Cu}$ status under two dominant land use systems, viz. irrigated and rainfed ecosystem of vellore district of Tamil Nadu using GPS and GIS techniques (Fig. 1).

\section{Materials and Methods}

One thousand three hundred and nineteen samples representing rainfed ecosystem were collected. The major crops cultivated under rainfed conditions were Groundnut, redgram, cowpea, sorghum, horse gram, cotton and ragi. Seven hundred and fourteen samples representing irrigated ecosystem were collected and major crops grown under irrigated situation were rice, sugarcane, groundnut, ragi, sorghum, coconut, maize, turmeric and lilly. The rainfed and irrigated ecosystem spread all over the blocks in Vellore district. The surface geo referenced soil samples were processed and analysed for
$\mathrm{pH}$, organic carbon, CEC, and available nitrogen, phosphorus and potassium following standard methods. The DTPA- extractable Fe, $\mathrm{Zn}, \mathrm{Mn}$ and $\mathrm{Cu}$ were extracted with diethelene tri- amine penta-acetic acid (DTPA) solution (Lindsay and Norwell 1978) and subsequently analysed with the help of atomic absorption spectrophotometer (Chemito-203).

\section{Results and Discussion}

The $\mathrm{pH}$ of the soils in irrigated system ranged from 6.25 to 8.82 with a mean of 7.64 (Table $2)$. The highest mean soil $\mathrm{pH}$ was found in K.V. Kuppam and Alangayan (7.76) followed by Thirupattur (7.75) block (Table 3). Almost all soils in irrigated condition were slightly alkaline in soil reaction. Generally the soils under rainfed condition had neutral to alkaline soil $\mathrm{pH}$ (7.89) with a range of 6.32 to 8.81 (Table 2). The highest mean is noticed in Natrampally (7.74) block and the lowest is noticed in Gudiyattam (7.39) block. The overall mean electrical conductivity in the soils of Vellore district in irrigated environment showed low salt content $(0.27 \mathrm{dS}$ $\mathrm{m}^{-1}$ ) (Table 2). The highest electrical conductivity of the soils under irrigated system was recorded in Nemili $\left(0.33 \mathrm{dS} \mathrm{m}^{-1}\right)$ block while the lowest in Gudiyattam block $\left(0.20 \mathrm{dS} \mathrm{m}^{-1}\right)$ (Table 3). The EC of the rainfed soils ranged from 0.10 to $0.82 \mathrm{dS} \mathrm{m}^{-1}$ with a mean of 0.28 indicating low in salt concentration (Table 2). The highest mean electrical conductivity is noticed in Kaniymbadi $\left(0.34 \mathrm{dS} \mathrm{m}^{-1}\right)$ and the lowest mean EC is noticed in Arcot, thirupatthur, Madhanur, Anaicut and Walajapet (0.26 dS $\left.\mathrm{m}^{-1}\right)$. The organic carbon content of the soils under irrigated system ranged from 0.18 to 0.70 per cent with a mean of 0.42 per cent revealing low in organic carbon status of the soils (Table 2). The highest mean organic carbon content was recorded in Natrampally and Kaniyambadi block ( 0.47 per cent) while 
the lowest in Alangayan ( 0.35 per cent) block (Table 3). The mean organic carbon content of the soils under rainfed situation was low ( 0.43 percent) in fertility and ranged from 0.15 to 0.85 per cent (Table 2). Highest organic carbon status ( 0.47 per cent) was found in Gudiyattam block and the lowest in Arakkonam (0.35 per cent) block (Table 5). The free $\mathrm{CaCO}_{3}$ (per cent) content of the soils in irrigated system showed a mean of 2.9 per cent indicating non calcareous nature of soils (Table 2). The highest mean free $\mathrm{CaCO}_{3}$ content was noted in the soils of Arcot (4.0 per cent) block and the lowest in Alangayan (1.4 per cent) block (Table 2). The mean free $\mathrm{CaCO}_{3}$ content of the soils in rainfed cultivation system was non calcareous in nature and ranged from 0.13 to 13.9 per cent with a mean of 2.8 per cent (Table 2). The highest mean free $\mathrm{CaCO}_{3}$ registered in the soils of Natrampally block (4.0 per cent) while the lowest in Alangayan and Anaicut (2.20 per cent) block soils (Table 5).

Micronutrient availability in the soils of Vellore district under irrigated situation was observed with mean of $42.6 \mathrm{mg} \mathrm{kg}^{-1}, 28.4 \mathrm{mg}$ $\mathrm{kg}^{-1}, 1.8 \mathrm{mg} \mathrm{kg}^{-1}$ and $2.4 \mathrm{mg} \mathrm{kg}^{-1}$ for available iron, manganese, zinc and copper respectively (Table 2). Considering the critical limits 1.2 $\mathrm{mg} \mathrm{kg}{ }^{-1}$ for available $\mathrm{Zn}, 0.2 \mathrm{mg} \mathrm{kg}^{-1}$ for available $\mathrm{Cu}, 4.2 \mathrm{mg} \mathrm{kg}^{-1}$ for available $\mathrm{Fe}, 2.0$ $\mathrm{mg} \mathrm{kg}^{-1}$ for available $\mathrm{Mn}$ as proposed by Lindsay and Norvell (1978), the deficient range of $\mathrm{Zn}$ noticed under irrigated condition in Jolarpet, Natrampally, Thirupattur and madhanur blocks of vellore district (Table 4).

The DTPA-Zn content in the soils of irrigated system ranged from 0.20 to $11.1 \mathrm{mg} \mathrm{kg}^{-1}$ with a mean of $1.8 \mathrm{mg} \mathrm{kg}^{-1}$ and the highest mean $\mathrm{Zn}$ content was found in walajapet block (3.7 $\mathrm{mg} \mathrm{kg}^{-1}$ ) and the lowest mean $\mathrm{Zn}$ content was found in Madhanur $\left(0.7 \mathrm{mg} \mathrm{kg}^{-1}\right)$ block (Table 4). All the blocks had higher DTPA-Fe content and values ranged from 5.5 to 98.1 $\mathrm{mg} \mathrm{kg}^{-1}$ (Table 4). The soils of vellore block recorded the highest mean $\mathrm{Fe}$ availability $\left(80.6 \mathrm{mg} \mathrm{kg}^{-1}\right.$ ) while the lowest Fe status in Walajapet block $\left(28.5 \mathrm{mg} \quad \mathrm{kg}^{-1}\right)$ under irrigated condition (Table 4). The sufficient range of $\mathrm{Cu}$ noticed in all the blocks of Vellore district (Table 4) under irrigated system. The DTPA-Cu content in the soils of irrigated system ranged from 0.3 to $7.4 \mathrm{mg}$ $\mathrm{kg}-1$ with overall mean of $2.4 \mathrm{mg} \mathrm{kg}^{-1}$ (Table 4). The highest mean $\mathrm{Cu}$ content in the soils was found in Alangayan block (3.9 $\mathrm{mg} \mathrm{kg}^{-1}$ ) and the lowest in Peranambattu (1.4 $\left.\mathrm{mg} \mathrm{kg}^{-1}\right)$ blocks (Table 4). The DTPA-Mn content in the soils under irrigated condition ranged from 1.2 to $67.8 \mathrm{mg} \mathrm{kg}^{-1}$ with a mean of 28.4 $\mathrm{mg} \mathrm{kg}^{-1}$ and the highest mean Mn availability was noted in the soils of Gudiyattam block (62.5 $\mathrm{mg} \mathrm{kg}^{-1}$ ) whereas the lowest mean Mn content was in Arakkonam block $\left(8.4 \mathrm{mg} \mathrm{kg}^{-}\right.$ $\left.{ }^{1}\right)$ (Table 4). Considering the critical limits of $2.0 \mathrm{mg} \mathrm{kg}{ }^{-1}$, proposed by Lindsay and Norvell (1978), the sufficient range of Mn noticed in all the blocks of Vellore district.

In general, soils under irrigated system, deficient range of $\mathrm{Zn}$ noticed in Jolarpet, Natrampally, Thirupattur and Madhanur blocks of Vellore district. The $\mathrm{Cu}$ was sufficient in all the blocks except in Vellore block. The Fe and Mn was sufficient in all the blocks. The soils under rainfed condition possessed lesser values of all the nutrients than irrigated soil environment except for $\mathrm{Zn}$ which is equal $\left(1.8 \mathrm{mg} \mathrm{kg}^{-1}\right)$. Deficient soil samples were found in all the blocks, for Fe, $\mathrm{Zn}, \mathrm{Mn}$ and $\mathrm{Cu}$ in district (Table 6). The DTPA-Zn content in the soils varied from 0.1 to $11.4 \mathrm{mg} \mathrm{kg}^{-1}$ with a mean of $1.80 \mathrm{mg} \mathrm{kg}^{-1}$. The highest mean DTPA Zn content noticed in the soils of Walajapet block $\left(3.5 \mathrm{mg} \mathrm{kg}^{-1}\right)$ and the lowest $\mathrm{Zn}$ status in Thiruppathur (0.5 $\mathrm{mg} \mathrm{kg}{ }^{-1}$ ) block soils under rainfed system (Table 6). Considering the critical limit, Arakkonam, Jolarpet, Thiruppathur and Madhanur blocks have deficient range of $\mathrm{Zn}$. 
Fig.1 Location map of Vellore district

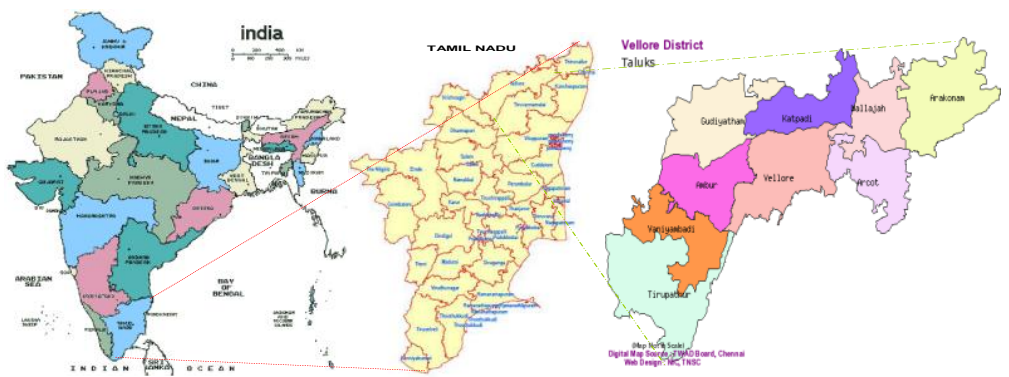

Table.1 Details of geo referenced soil samples

\begin{tabular}{|c|c|c|c|}
\hline S. No. & Taluk name & Block name & No. of samples \\
\hline \multirow[t]{3}{*}{1} & Arakkonam & 1. Arakkonam & 108 \\
\hline & & 2. Kaveripakkam & 224 \\
\hline & & 3. Nemili & 228 \\
\hline \multirow[t]{2}{*}{2} & Arcot & 4. Arcot & 163 \\
\hline & & 5. Timiri & 260 \\
\hline \multirow[t]{4}{*}{3} & Gudiyattam & 6. Gudiyattam & 172 \\
\hline & & 7. Katpadi & 82 \\
\hline & & 8. K.V.Kuppam & 152 \\
\hline & & 9. Peranambattu & 196 \\
\hline \multirow[t]{4}{*}{4} & Thiruppathur & 10. Jolarpet & 148 \\
\hline & & 11. Kandili & 156 \\
\hline & & 12. Natrampalli & 115 \\
\hline & & 13. Tiruppathur & 124 \\
\hline \multirow[t]{2}{*}{5} & Kaniyambadi & 14. Alangayan & 98 \\
\hline & & 15. Madhanur & 141 \\
\hline \multirow[t]{3}{*}{6} & Vellore & 16. Anaicut & 152 \\
\hline & & 17. Kaniyambadi & 104 \\
\hline & & 18. Vellore & 76 \\
\hline \multirow[t]{3}{*}{7} & Walajapet & 19. Sholinghur & 168 \\
\hline & & 20. Walajapet & 143 \\
\hline & & TOTAL & 3010 \\
\hline
\end{tabular}

Table.2 The data of soil properties and micronutrient status

\begin{tabular}{|l|l|l|l|l|}
\hline \multirow{2}{*}{ Parameter } & \multicolumn{2}{c|}{ Irrigated } & \multicolumn{2}{c|}{ Rainfed } \\
\cline { 2 - 5 } & Range & Mean & Range & Mean \\
\hline No. of samples & 714 & & 1319 \\
\hline $\mathrm{pH}$ & $6.25-8.82$ & 7.64 & $6.32-8.81$ & 7.55 \\
\hline $\left.\mathrm{EC}\left(\mathrm{dSm}^{-1}\right)\right)$ & $0.11-0.59$ & 0.27 & $0.10-0.82$ & 0.28 \\
\hline${\left.\mathrm{OC}\left(\mathrm{mg} \mathrm{k}^{-1}\right)\right)}^{1.80-7.90}$ & 4.20 & $1.50-8.50$ & 4.30 \\
\hline $\mathrm{CaCO}_{3}(\%)$ & $0.3-13.80$ & 2.90 & $0.13-13.9$ & 2.80 \\
\hline Micronutrient status $\left(\mathbf{m g ~ k g}^{-1}\right)$ & & \\
\hline DTPA-Zn & $0.2-11.1$ & 1.80 & $0.1-11.4$ & 1.80 \\
\hline DTPA-Fe & $5.5-98.1$ & 42.6 & $1.8-71.2$ & 13.8 \\
\hline DTPA-Cu & $0.3-7.40$ & 2.40 & $0.2-12.1$ & 2.20 \\
\hline DTPA-Mn & $1.2-67.8$ & 28.4 & $0.8-59.4$ & 18.0 \\
\hline
\end{tabular}


Table.3 Chemical properties of soil under irrigated condition in different blocks of Vellore district $(n=714)$

\begin{tabular}{|c|c|c|c|c|c|c|c|c|c|c|c|}
\hline \multirow[t]{2}{*}{ S. no } & \multirow{2}{*}{$\begin{array}{c}\text { Name of the } \\
\text { block }\end{array}$} & \multirow{2}{*}{$\begin{array}{c}\text { No. of } \\
\text { Samples }\end{array}$} & \multirow[t]{2}{*}{ Crops grown } & \multicolumn{2}{|c|}{ pH } & \multicolumn{2}{|c|}{$\operatorname{EC}\left(\mathrm{dSm}^{-1}\right)$} & \multicolumn{2}{|c|}{$\mathrm{OC}\left(\mathrm{mg} \mathrm{kg}^{-1}\right)$} & \multicolumn{2}{|c|}{ Free $\mathrm{CaCO}_{3}(\%)$} \\
\hline & & & & Range & Mean & Range & Mean & Range & Mean & Range & Mean \\
\hline 1 & Arakkonam & 80 & Rice, Sugarcane & $6.25-8.82$ & 7.66 & $0.11-0.57$ & 0.28 & $2.1-7.0$ & 4.2 & $0.9-10.6$ & 3.2 \\
\hline 2 & Kaveripakkam & 115 & Rice, Sugarcane & $6.29-8.75$ & 7.71 & $0.11-0.56$ & 0.30 & $1.9-7.9$ & 4.2 & $0.3-11.5$ & 3.8 \\
\hline 3 & Nemili & 169 & Rice, Lilly & $6.46-8.54$ & 7.66 & $0.12-0.57$ & 0.33 & $1.7-6.5$ & 3.9 & $0.9-11.3$ & 3.0 \\
\hline 4 & Arcot & 56 & Rice, Sugarcane. & $6.31-8.82$ & 7.73 & $0.15-0.58$ & 0.28 & $1.8-6.2$ & 4.5 & $1.1-13.8$ & 4.0 \\
\hline 5 & Timiri & 103 & $\begin{array}{l}\text { Rice, Sugarcane, } \\
\text { Groundnut }\end{array}$ & $6.49-8.82$ & 7.69 & $0.11-0.24$ & 0.31 & $1.8-7.2$ & 4.2 & $0.3-13.5$ & 3.5 \\
\hline 6 & Gudiyattam & 5 & Rice, Ragi. & $7.08-8.54$ & 7.70 & $0.11-0.30$ & 0.20 & $3.8-5.4$ & 4.6 & $1.3-2.9$ & 1.8 \\
\hline 7 & Katpadi & 14 & Rice, Sugarcane. & $6.92-8.34$ & 7.68 & $0.11-0.37$ & 0.26 & $2.5-5.2$ & 4.0 & $1.4-6.1$ & 2.4 \\
\hline 8 & K.V.Kuppam & 9 & Rice, Sorghum. & $6.94-8.54$ & 7.76 & $0.16-0.35$ & 0.25 & $1.9-5.8$ & 3.8 & $1.0-9.0$ & 3.9 \\
\hline 9 & Peranambattu & 8 & Rice, Sugarcane. & $6.58-8.35$ & 7.47 & $0.11-0.33$ & 0.24 & $1.6-5.4$ & 3.9 & $0.9-10.4$ & 2.8 \\
\hline 10 & Jolarpet & 11 & Rice, Ragi. & $7.11-8.24$ & 7.67 & $0.18-0.46$ & 0.27 & $3.4-5.4$ & 4.0 & $0.9-2.8$ & 1.8 \\
\hline 11 & Kandili & 12 & Rice, Ragi, Coconut. & $7.23-8.41$ & 7.68 & $0.15-0.41$ & 0.28 & $3.0-5.9$ & 4.3 & $1.4-8.1$ & 2.8 \\
\hline 12 & Natrampalli & 3 & Rice, & $6.92-8.24$ & 7.57 & $0.15-0.35$ & 0.28 & $3.9-5.9$ & 4.7 & $1.3-3.5$ & 2.1 \\
\hline 13 & Tiruppathur & 12 & Rice, Maize. & $6.48-8.56$ & 7.75 & $0.12-0.59$ & 0.30 & $2.5-5.6$ & 4.2 & $1.0-8.6$ & 3.2 \\
\hline 14 & Alangayan & 2 & Rice, & $7.54-7.98$ & 7.76 & $0.24-0.31$ & 0.28 & $2.8-4.2$ & 3.5 & $1.4-1.5$ & 1.4 \\
\hline 15 & Madhanur & 7 & Rice, Ragi. & $6.84-7.91$ & 7.44 & $0.16-0.29$ & 0.23 & $3.0-5.5$ & 4.0 & $1.3-2.9$ & 2.0 \\
\hline 16 & Anaicut & 7 & Rice, Sugarcane. & $6.99-7.93$ & 7.55 & $0.15-0.31$ & 0.21 & $3.4-7.3$ & 4.1 & $1.1-11.8$ & 2.8 \\
\hline 17 & Kaniyambadi & 10 & Rice, Turmeric. & $6.94-8.55$ & 7.70 & $0.16-0.35$ & 0.26 & $3.8-6.4$ & 4.7 & $1.1-10.6$ & 3.7 \\
\hline 18 & Vellore & 3 & Rice, & $7.28-7.68$ & 7.47 & $0.24-0.31$ & 0.28 & $2.8-5.4$ & 3.7 & $3.0-3.8$ & 3.3 \\
\hline 19 & Sholinghur & 40 & $\begin{array}{l}\text { Rice, Ragi, } \\
\text { Sugarcane. }\end{array}$ & $6.57-8.49$ & 7.51 & $0.13-0.47$ & 0.28 & $1.9-6.8$ & 4.2 & $0.9-11.3$ & 3.7 \\
\hline 20 & Walajapet & 48 & $\begin{array}{l}\text { Rice, Groundnut, } \\
\text { Sugarcane }\end{array}$ & $6.48-8.48$ & 7.56 & $0.11-0.45$ & 0.28 & $1.9-7.4$ & 4.6 & $0.3-10.4$ & 2.8 \\
\hline \multicolumn{2}{|c|}{ District } & 714 & & $6.25-8.82$ & 7.64 & $0.11-0.59$ & 0.27 & $1.8-7.9$ & 4.2 & $0.3-13.8$ & 2.9 \\
\hline
\end{tabular}

Table.4 Micronutrient availability in the soil under irrigated condition in different blocks of Vellore district $(n=714)$

\begin{tabular}{|c|c|c|c|c|c|c|c|c|c|c|}
\hline \multirow[t]{2}{*}{ S. No } & \multirow{2}{*}{$\begin{array}{l}\text { Name of the } \\
\text { block }\end{array}$} & \multirow{2}{*}{$\begin{array}{l}\text { No. of } \\
\text { Sample }\end{array}$} & \multicolumn{2}{|c|}{$\mathrm{Fe}\left(\mathrm{mgkg}^{-1}\right)$} & \multicolumn{2}{|c|}{ Mn $\left(\mathrm{mgkg}^{-1}\right)$} & \multicolumn{2}{|c|}{ Zn $\left(\mathrm{mgkg}^{-1}\right)$} & \multicolumn{2}{|c|}{$\mathrm{Cu}\left(\mathrm{mgkg}^{-1}\right)$} \\
\hline & & & Range & Mean & Range & Mean & Range & Mean & Range & Mean \\
\hline 1 & Arakkonam & 80 & $10.7-82.9$ & 33.5 & $1.2-21.0$ & 8.4 & $0.4-4.9$ & 1.2 & $0.3-6.6$ & 2.3 \\
\hline 2 & Kaveripakkam & 115 & $8.8-98.1$ & 52.4 & $2.2-67.8$ & 33.8 & $0.2-6.7$ & 1.6 & $0.4-5.8$ & 1.7 \\
\hline 3 & Nemili & 169 & $5.5-87.8$ & 33.3 & $1.2-64.2$ & 20.8 & $0.4-7.7$ & 1.7 & $0.4-7.4$ & 1.8 \\
\hline 4 & Arcot & 56 & $43.5-85.9$ & 63.2 & $8.9-43.7$ & 29.9 & $0.3-11.1$ & 1.8 & $0.4-4.4$ & 2.2 \\
\hline 5 & Timiri & 103 & $26.0-70.3$ & 40.8 & $7.9-40.7$ & 30.8 & $0.4-7.5$ & 1.7 & $0.6-7.3$ & 2.3 \\
\hline 6 & Gudiyattam & 5 & $49.2-56.6$ & 53.0 & $60.5-66.0$ & 62.5 & $1.2-3.5$ & 2.0 & $1.2-1.9$ & 1.6 \\
\hline 7 & Katpadi & 14 & $28.0-49.5$ & 34.3 & $10.1-23.5$ & 14.4 & $0.8-3.3$ & 1.7 & $1.8-6.8$ & 3.2 \\
\hline 8 & K.V.Kuppam & 9 & $24.4-41.1$ & 29.9 & $27.5-38.3$ & 31.2 & $1.1-7.5$ & 2.9 & $1.1-7.1$ & 3.8 \\
\hline 9 & Peranambattu & 8 & $32.8-56.5$ & 39.7 & $45.7-49.4$ & 47.4 & $0.5-8.0$ & 2.5 & $0.7-2.62$ & 1.4 \\
\hline 10 & Jolarpet & 11 & $33.6-63.2$ & 48.1 & $13.3-26.8$ & 18.3 & $0.4-1.4$ & 0.9 & $2.6-5.8$ & 3.4 \\
\hline 11 & Kandili & 12 & $22.0-44.0$ & 34.0 & $17.3-29.7$ & 21.0 & $1.1-9.6$ & 3.2 & $1.2-5.2$ & 3.0 \\
\hline 12 & Natrampalli & 3 & $29.2-64.7$ & 41.4 & $15.9-16.9$ & 16.3 & $0.6-1.1$ & 1.0 & $0.9-2.8$ & 1.8 \\
\hline 13 & Tiruppathur & 12 & $26.4-47.4$ & 39.4 & $24.8-46.6$ & 30.5 & $0.2-1.7$ & 0.6 & $0.7-6.5$ & 3.5 \\
\hline 14 & Alangayan & 2 & $32.7-37.6$ & 35.2 & $19.1-20.3$ & 19.7 & $1.3-2.7$ & 2.0 & $3.1-4.8$ & 3.9 \\
\hline 15 & Madhanur & 7 & $31.2-36.2$ & 33.8 & $51.3-54.4$ & 52.9 & $0.4-1.3$ & 0.7 & $1.3-2.9$ & 2.0 \\
\hline 16 & Anaicut & 7 & $28.8-44.2$ & 38.0 & $39.4-50.8$ & 45.8 & $0.9-1.9$ & 1.4 & $0.8-3.2$ & 1.9 \\
\hline 17 & Kaniyambadi & 10 & $38.6-54.4$ & 42.7 & $8.2-38.3$ & 22.5 & $0.7-4.4$ & 2.1 & $1.6-5.8$ & 3.2 \\
\hline 18 & Vellore & 3 & $74.4-89.2$ & 80.6 & $6.8-33.0$ & 22.5 & $0.8-1.9$ & 1.2 & $0.6-1.1$ & 0.9 \\
\hline 19 & Sholinghur & 40 & $25.9-75.0$ & 50.5 & $2.3-40.5$ & 24.5 & $0.3-4.4$ & 1.3 & $0.4-5.7$ & 2.1 \\
\hline 20 & Walajapet & 48 & $18.8-57.4$ & 28.5 & $7.4-35.8$ & 13.9 & $0.2-9.7$ & 3.7 & $0.4-4.0$ & 1.8 \\
\hline \multicolumn{2}{|c|}{ District } & 714 & $5.5-98.1$ & 42.6 & $1.2-67.8$ & 28.4 & $0.2-11.1$ & 1.8 & $0.3-7.4$ & 2.4 \\
\hline
\end{tabular}


Table.5 Chemical properties of soil under rainfed condition in different blocks of Vellore district

$$
(n=1319)
$$

\begin{tabular}{|c|c|c|c|c|c|c|c|c|c|c|c|}
\hline \multirow{2}{*}{$\begin{array}{l}\text { S. } \\
\text { No }\end{array}$} & \multirow{2}{*}{$\begin{array}{l}\text { Name of the } \\
\text { block }\end{array}$} & \multirow{2}{*}{$\begin{array}{l}\text { No. of } \\
\text { samples }\end{array}$} & \multirow[t]{2}{*}{ Crops Grown } & \multicolumn{2}{|c|}{$\mathrm{pH}$} & \multicolumn{2}{|c|}{$\operatorname{EC}\left(d_{S m^{-1}}\right)$} & \multicolumn{2}{|c|}{$\mathrm{OC}\left(\mathrm{mg} \mathrm{kg}^{-1}\right)$} & \multicolumn{2}{|c|}{ Free $\mathrm{CaCO}_{3}(\%)$} \\
\hline & & & & Range & Mean & Range & Mean & Range & Mean & Range & Mean \\
\hline 1 & Arakkonam & 5 & Groundnut & $7.28-7.64$ & 7.43 & $0.23-0.41$ & 0.32 & $2.7-4.3$ & 3.5 & $1.1-3.1$ & 2.3 \\
\hline 2 & Kaveripakkam & 45 & Cowpea, Groundnut & $6.61-8.54$ & 7.66 & $0.11-0.50$ & 0.27 & $2.4-7.1$ & 4.3 & $1.0-9.5$ & 2.7 \\
\hline 3 & Nemili & 13 & Cowpea, Groundnut & $6.52-7.91$ & 7.47 & $0.16-0.42$ & 0.27 & $2.4-6.2$ & 4.4 & $1.3-10.4$ & 3.5 \\
\hline 4 & Arcot & 50 & Redgram, Groundnut & $6.89-8.66$ & 7.73 & $0.11-0.55$ & 0.26 & $2.2-6.2$ & 4.1 & $0.3-11.8$ & 3.3 \\
\hline 5 & Timiri & 63 & $\begin{array}{l}\text { Groundnut, Red gram, } \\
\text { Sorghum }\end{array}$ & $6.57-8.57$ & 7.63 & $0.11-0.61$ & 0.30 & $2.4-7.1$ & 4.4 & $0.3-11.5$ & 2.9 \\
\hline 6 & Gudiyattam & 112 & $\begin{array}{l}\text { Ragi, Redgram, } \\
\text { Horsegram, Sorghum }\end{array}$ & $6.48-8.56$ & 7.39 & $0.10-0.58$ & 0.28 & $2.2-7.8$ & 4.7 & $0.1-12.5$ & 2.5 \\
\hline 7 & Katpadi & 41 & $\begin{array}{l}\text { Redgram, Sorghum, } \\
\text { Horsegram }\end{array}$ & $6.36-8.46$ & 7.59 & $0.16-0.56$ & 0.30 & $2.1-5.9$ & 4.1 & $0.3-10.6$ & 2.8 \\
\hline 8 & K.V.Kuppam & 100 & $\begin{array}{l}\text { Ragi, Horsegram, } \\
\text { Sorghum }\end{array}$ & $6.54-8.56$ & 7.51 & $0.11-0.59$ & 0.28 & $1.6-7.7$ & 4.3 & $0.9-10.3$ & 3.0 \\
\hline 9 & Peranambattu & 140 & Ragi, Sorghum & $6.32-8.58$ & 7.43 & $0.11-0.54$ & 0.27 & $2.3-7.8$ & 4.6 & $0.9-10.4$ & 2.3 \\
\hline 10 & Jolarpet & 93 & Cotton, Ragi, Sorghum & $6.34-8.54$ & 7.6 & $0.1-0.24$ & 0.27 & $1.6-7.7$ & 4.6 & $0.4-10.8$ & 3.0 \\
\hline 11 & Kandili & 114 & $\begin{array}{l}\text { Cotton, Ragi, Redgram, } \\
\text { Sorghum }\end{array}$ & $6.79-8.53$ & 7.61 & $0.11-0.19$ & 0.27 & $2.4-6.5$ & 4.2 & $0.4-13.4$ & 2.9 \\
\hline 12 & Natrampalli & 93 & Cotton, Ragi, Sorghum & $6.78-8.59$ & 7.74 & $0.11-0.57$ & 0.31 & $1.8-6.2$ & 3.8 & $0.9-10.8$ & 4.0 \\
\hline 13 & Tiruppathur & 58 & Cotton, Redgram, Ragi. & $6.33-8.63$ & 7.55 & $0.11-0.82$ & 0.26 & $1.9-7.4$ & 4.2 & $0.9-13.9$ & 3.3 \\
\hline 14 & Alangayan & 57 & Cotton, Ragi, Sorghum & $6.36-8.48$ & 7.54 & $0.11-0.45$ & 0.27 & $2.5-8.5$ & 4.3 & $0.9-7.9$ & 2.2 \\
\hline 15 & Madhanur & 62 & Groundnut, Sorghum & $6.49-8.48$ & 7.45 & $0.11-0.53$ & 0.26 & $1.9-8.3$ & 4.3 & $1.0-13.3$ & 2.6 \\
\hline 16 & Anaicut & 70 & Ragi, Sorghum & $6.35-8.48$ & 7.43 & $0.12-0.45$ & 0.26 & $1.5-6.2$ & 4.0 & $0.1-12.4$ & 2.2 \\
\hline 17 & Kaniyambadi & 56 & $\begin{array}{l}\text { Groundnut, Redgram, } \\
\text { Sorghum }\end{array}$ & $6.37-8.48$ & 7.67 & $0.12-0.26$ & 0.34 & $1.9-7.4$ & 4.3 & $0.6-10.9$ & 2.9 \\
\hline 18 & Vellore & 62 & Groundnut, Sorghum & $6.54-8.53$ & 7.60 & $0.13-0.60$ & 0.3 & $1.6-6.2$ & 4.1 & $1.0-10.6$ & 2.7 \\
\hline 19 & Sholinghur & 47 & $\begin{array}{l}\text { Groundnut, Redgram, } \\
\text { Sorghum }\end{array}$ & $6.54-8.81$ & 7.53 & $0.11-0.43$ & 0.28 & $1.9-7.4$ & 4.5 & $0.9-13.4$ & 3.2 \\
\hline 20 & Walajapet & 38 & $\begin{array}{l}\text { Groundnut, Redgram, } \\
\text { Sorghum }\end{array}$ & $6.54-8.48$ & 7.52 & $0.14-0.54$ & 0.26 & $2.5-7.4$ & 4.4 & $0.5-10.9$ & 2.8 \\
\hline & District & 1319 & & $6.32-8.81$ & 7.55 & $0.10-0.82$ & 0.28 & $1.5-8.5$ & 4.3 & $0.1-13.9$ & 2.8 \\
\hline
\end{tabular}

Table.6 Micronutrient availability in the soil under rainfed condition in different blocks of Vellore district $(n=1319)$

\begin{tabular}{|c|c|c|c|c|c|c|c|c|c|c|}
\hline \multirow[t]{2}{*}{ S. No } & \multirow{2}{*}{$\begin{array}{l}\text { Name of the } \\
\text { block }\end{array}$} & \multirow{2}{*}{$\begin{array}{l}\text { No. of } \\
\text { sample }\end{array}$} & \multicolumn{2}{|c|}{$\mathrm{Fe}\left(\mathrm{mgkg}^{-1}\right)$} & \multicolumn{2}{|c|}{$\operatorname{Mn}\left(\mathrm{mgkg}^{-1}\right)$} & \multicolumn{2}{|c|}{ Zn $\left(\mathrm{mgkg}^{-1}\right)$} & \multicolumn{2}{|c|}{$\mathrm{Cu}\left(\mathrm{mgkg}^{-1}\right)$} \\
\hline & & & Range & Mean & Range & Mean & Range & Mean & Range & Mean \\
\hline 1 & Arakkonam & 5 & $6.9-9.0$ & 8.0 & $3.1-17.9$ & 7.1 & $0.3-1.2$ & 0.7 & $0.9-4.9$ & 2.1 \\
\hline 2 & Kaveripakkam & 45 & $3.0-25.0$ & 13.0 & $2.2-45.5$ & 19.5 & $0.3-4.4$ & 1.4 & $0.2-2.7$ & 1.2 \\
\hline 3 & Nemili & 13 & $6.0-13.8$ & 9.1 & $2.0-25.1$ & 13.2 & $0.8-6.9$ & 2.2 & $0.4-3.9$ & 1.4 \\
\hline 4 & Arcot & 50 & $4.8-44.0$ & 28.6 & $8.4-41.5$ & 29.1 & $0.4-7.1$ & 1.8 & $0.4-4.0$ & 2.0 \\
\hline 5 & Timiri & 63 & $2.6-27.5$ & 13.8 & $0.8-39.5$ & 25.3 & $0.3-4.5$ & 1.3 & $0.6-4.8$ & 1.9 \\
\hline 6 & Gudiyattam & 112 & $1.8-20.5$ & 11.2 & $0.9-59.4$ & 30.8 & $0.4-8.0$ & 2.8 & $0.9-4.0$ & 1.8 \\
\hline 7 & Katpadi & 41 & $4.3-27.8$ & 13.1 & $1.5-9.6$ & 4.1 & $0.5-5.8$ & 1.6 & $1.1-6.6$ & 2.7 \\
\hline 8 & K.V.Kuppam & 100 & $4.6-19.2$ & 9.8 & $3.5-26.5$ & 12.2 & $0.7-9.4$ & 2.0 & $0.8-8.1$ & 2.8 \\
\hline 9 & Peranambattu & 140 & $3.3-22.4$ & 10.5 & $7.5-47.1$ & 26.6 & $0.1-7.6$ & 2.1 & $0.5-3.7$ & 1.3 \\
\hline 10 & Jolarpet & 93 & $5.4-21.6$ & 14.5 & $1.5-13.2$ & 6.0 & $0.2-3.9$ & 1.0 & $0.8-12.1$ & 3.0 \\
\hline 11 & Kandili & 114 & $6.4-21.9$ & 15.0 & $1.7-19.1$ & 10.2 & $0.6-6.5$ & 2.1 & $0.4-9.8$ & 3.3 \\
\hline 12 & Natrampalli & 93 & $4.4-20.6$ & 13.0 & $0.6-16.2$ & 13.7 & $0.2-7.0$ & 2.1 & $0.7-5.6$ & 2.7 \\
\hline 13 & Tiruppathur & 58 & $3.5-14.1$ & 8.7 & $5.1-19.9$ & 13.8 & $0.2-1.2$ & 0.5 & $0.4-5.2$ & 2.2 \\
\hline 14 & Alangayan & 57 & $5.1-16.8$ & 12.8 & $1.6-19.0$ & 13.1 & $0.7-11.4$ & 2.1 & $1.4-5.7$ & 3.4 \\
\hline 15 & Madhanur & 62 & $2.3-13.4$ & 6.9 & $2.5-58.7$ & 37.6 & $0.3-4.1$ & 0.9 & $0.9-4.4$ & 2.1 \\
\hline 16 & Anaicut & 70 & $4.3-26.7$ & 17.4 & $11.0-52.5$ & 45.2 & $0.4-3.9$ & 1.2 & $1.0-4.8$ & 2.2 \\
\hline 17 & Kaniyambadi & 56 & $3.6-37.6$ & 19.7 & $2.6-39.0$ & 20.6 & $0.6-4.6$ & 1.9 & $0.3-4.9$ & 2.4 \\
\hline 18 & Vellore & 62 & $6.9-71.2$ & 31.3 & $1.8-33.2$ & 9.4 & $0.1-7.9$ & 2.4 & $0.5-8.0$ & 1.7 \\
\hline 19 & Sholinghur & 47 & $3.3-23.1$ & 10.2 & $2.8-39.4$ & 18.1 & $0.4-5.4$ & 1.6 & $0.4-5.1$ & 1.4 \\
\hline \multirow[t]{2}{*}{20} & Walajapet & 38 & $3.5-18.1$ & 10.1 & $0.8-8.4$ & 3.9 & $0.1-9.3$ & 3.5 & $0.6-3.7$ & 1.6 \\
\hline & District & 1319 & $1.8-71.2$ & 13.8 & $0.8-59.4$ & 18.0 & $0.1-11.4$ & 1.8 & $0.2-12.1$ & 2.2 \\
\hline
\end{tabular}

The overall mean DTPA-Fe availability in the soils of rainfed system was $13.8 \mathrm{mg} \mathrm{kg}-1$ with a range of 1.8 to $71.2 \mathrm{mg} \mathrm{kg-1revealing} \mathrm{very}$ high availability of Fe. The highest mean Fe availability recorded in Vellore $\left(31.3 \mathrm{mg} \mathrm{kg}^{-1}\right)$ block while the lowest was in Madhanur (6.9 
mg kg-1) block under rainfed system (Table 6). The DTPA $\mathrm{Cu}$ content in the soils of rainfed system varied from 0.2 to $12.1 \mathrm{mg} \mathrm{kg}$ ${ }^{1}$ and the highest mean $\mathrm{Cu}$ content found in Alangayan (3.4 mg kg-1). The lesser DTPA $\mathrm{Cu}$ status, noticed in Kaveripakkam $(1.2 \mathrm{mg}$ $\mathrm{kg}^{-1}$ ) block (Table 6). The DTPA-Mn in the rainfed soils ranged from 0.8 to $59.4 \mathrm{mg} \mathrm{kg}^{-1}$ with a mean value of $18.0 \mathrm{mg} \mathrm{kg}$. The highest mean Mn status registered in Anaicut block (45.2 mg kg-1) whereas the lowest in Walajapet (3.9 $\mathrm{mg} \mathrm{kg}^{-1}$ ) (Table 6).

To conclude that, there is no wide variation in the soil properties under both irrigated and rainfed systems (Table 1). However the higher soil $\mathrm{pH}$ and Free $\mathrm{CaCO}_{3}$ was noticed in Irrigated system. The rainfed system had higher amount of electrical conductivity than irrigated system. With regard to mean viability of micronutrient content, the irrigated environment registered the highest availability of all nutrients except $\mathrm{Zn}$ content, which was equal with rainfed condition.

Moisture plays an important role in soil nutrient availability by influencing the soil chemical characteristics. "slightly acidic to alkaline" soil reaction was observed in both irrigated and rainfed situations and neutral $\mathrm{pH}$ under irrigated situations might be due to reduced condition prevailing under wetting (Ponnamperuma, 1972). The overall mean values of the soils indicated alkaline soil reaction, low salinity, low organic carbon and non-calcareous nature. However the range values showed a variation in organic carbon, $\mathrm{EC}$ and free $\mathrm{CaCO}_{3}$. Alkaline soil reaction, the higher $\mathrm{EC}$ values, $\mathrm{CaCO}_{3}(2.8 \%)$ were noticed in soils under rainfed system than irrigated system which were similar with the results from the studies done by Sharma et al., (2011) The soils under rainfed conditions had higher organic carbon which can be explained by increased $\mathrm{C}$ input through root biomass and similar results were reported by Singh et al., (2009). Lesser free $\mathrm{CaCO}_{3}$ content was observed under rainfed situation which might be due to deficit moisture availability and faster decomposition and loss (Datta et al., 1989). Although the nutrient availability was lesser in soils under rainfed situation, the mean values indicated sufficient nutrients availability in the soils of Vellore district except $\mathrm{Zn}$ which showed medium status under rainfed and irrigated conditions. Comparing the irrigated and rainfed situations, more than 65 per cent reduction in $\mathrm{Fe}$ and 37 per cent reduction in $\mathrm{Mn}$ and 8 per cent reduction in $\mathrm{Cu}$ availability was noted in rainfed soils and the order of reduction was higher $\mathrm{Fe}>\mathrm{Mn}>$ $\mathrm{Cu}$. Reduced water availability under rainfed situation leads to reduced solubilisation of nutrients to the available pool thus leads to reduced nutrient availability. Similar results were reported by Paramasivam et al., (1994). Higher availability of nutrients viz., $\mathrm{Fe}, \mathrm{Cu}$ and $\mathrm{Mn}$ in irrigated situation might be primarily attributed to the higher amount of organic residues produced and returned to the soil, increased nutrient solubilisation in the presence of moisture and reduced rate of organic matter decomposition in cultivated soils (Bayala et al., 2007; Mages and Nicholas 2008; Hundal et al., 2009). Reduced water availability under rainfed situation leads to reduced solubilisation of nutrients to the available pool thus leads to reduced nutrient availability. Similar results were reported by Pal et al., (2000) and Ajit Kumar et al., (2009).

\section{References}

Ajit Kumar, K., Singh, K. and Singh, R.N. (2009) Available nutrients and response of lentil (Lense esculenta) to boron application in rainfed uplands soils of Ranchi. Journal of the Indian Society of Soil Science 57(2), 219-222.

Bayala, J., Balesdent, J., Marol, C., Zapata, F., Teklehaimanot, Z. and Ouedraogo, 
S.O. (2007) Relative contribution of trees and crops to soil organic carbon content in a park land system in Burkina Faso. Advances in Integrated soil fertility management in Sub-Saharan Africa: Challenges and opportunities, 161-169.

Datta, M. and Ram, M. 1989. Status of micronutrients in some soil series of Tripura. Journal of the Indian Society of Soil Science, 41, 776-777.

Katyal, J.C. and Rattan, R.K. 2003. Secondary and micronutrients research gaps and future needs. Fertilizer News, 48, 9-14.

Lindsay, N.L. and Norvell, W.A. 1978. Development of DTPA soil test for zinc, iron, manganese and copper. Soil Science Society of America, 42, 421428.

Pal, S., Datta, S. P., Rattan, R. K. and Singh, A.K. (2000) Diagnosis and Amelioration of Iron Deficiency under Aerobic Rice. Journal of Plant Nutrition 31(5), 919 -940.

Paramasivam, P. and Gopalswamy, A. 1994. Distribution of micronutrients in Lower Bhavani Project (LBP) command area soil profiles. Madras Agricultural Journal, 81, 545- 547.

Ponnamperuma, F.N. 1972. The chemistry of submerged soils. Advances in Agronomy, 24, 29-96.

Rattan, R.K., Patel K.P., Manjaiah K.M. and Datta S.P. 2009. Micronutrients in soil, plant, animal and human health. Journal of the Indian Society of Soil Science, 47(4), 546- 558.
Rego, T.J., Sahrawat, K.L. Wani, S.P. Pardhasaradhi, G. 2007. Widespread deficiencies of sulphur, boron and zinc in dryland soils of the Indian semi arid tropical soils: on farm crop responses. Journal of Plant Nutrition 30, 15691583.

Sharma, J.C. and Chaudhary, S.K. 2007. Vertical distribution of micronutrient cations in relation to soil characteristics in lower Shiwaliks of Solan district in North-West Himalayas. Journal of the Indian Society of Soil Science, 55, 4044.

Sharma, R.C., Mandal, A.K. and Ranbir Singh. (2011) Delineation and Characterization of Waterlogged and Salt-affected Soils in Gandak Command Area of Bihar for Reclamation and Management. Journal of the Indian Society of Soil Science 59(4), 315-320.

Singh, K. 2009. The critical zinc deficiency levels in Indian soils and cereal crops. In: Proc. of the International Plant Nutritional Colloquium. XVI. Dept. of Plant Sciences, UC Davis.

Somasundaram, J., Singh, R. K., Paraldiyal, A. R. and Prasad, S. N. 2009. Micronutrient status of soils under different land use systems in Chambal ravines. Journal of the Indian Society of Soil Science, 57(3), 307-312.

Venkatesh, M. S., Majumdar, B., Kumar, K. and Patiram, 2003. Status of micronutrient cations under various land use systems of Meghalaya. Journal of Indian Society of Soil Science, 51, 6064.

\section{How to cite this article:}

Purma Soniya, D. Muthumanickam, M.R. Reshma and Jayasoorian, N. 2019. Status of DTPAExtractable Cationic Micronutrients and Physio Chemical Properties of Geo Referenced Soils Under Irrigated and Rainfed Ecosystems of Vellore District in Tamilnadu, India. Int.J.Curr.Microbiol.App.Sci. 8(06): 1590-1597. doi: https://doi.org/10.20546/ijcmas.2019.806.191 